\title{
Effects of long-range links on metastable states in a dynamic interaction network
}

\author{
Suhan Ree* \\ Center for Complex Quantum Systems and Department of Physics, University of Texas at Austin, Austin, Texas 78712, USA and \\ School of Liberal Arts and Science, Kongju National University, Yesan-Up, Yesan-Gun, Chungnam, 340-702, South Korea
}

(Received 2 September 2011; revised manuscript received 30 January 2012; published 20 April 2012)

\begin{abstract}
We introduce a model for random-walking agents on a two-dimensional periodic lattice, where the dynamic interaction network is defined using local short-range interactions and $E$ randomly added long-range interactions. With periodic states for agents and an interaction rule of repeated averaging, we numerically find two types of metastable states at low- and high- $E$ limits, respectively, along with consensus states. If we apply this model to opinion dynamics, metastable states can be interpreted as sustainable diversities in our societies, and our results then imply that, while diversities decrease and eventually disappear with more long-range interactions, other types of diverse states can appear under this rule when networks are almost fully connected.
\end{abstract}

DOI: 10.1103/PhysRevE.85.045101

PACS number(s): 89.75.Fb, 05.45.Xt, 87.23.Ge, 89.75.Hc

Complex systems usually consist of interconnected parts, forming complex networks, and typically exhibit complex and unpredictable behavior. While the concept of complex networks can be applied to various research areas, physicists have been trying to find general frameworks for the network theory [1-3], and their efforts can be summed up in three categories. First, generating mechanisms of static networks satisfying certain statistical properties as in small-world (SW) and scale-free networks [4-6], and statistical measures on their structures have been studied. Second, if nodes have states, the dynamic behavior of nodes on given static networks has been studied: for example, opinion dynamics [7-10] and synchronization problems $[11,12]$ on SW networks. Finally, the dynamic behavior of both nodes and links for "coevolving" networks [13-15], where nodes and links evolve, influencing each other, has been studied, especially when time scales for node and link dynamics are comparable.

Here we present a simple model of social networks with mobile agents [14,16-18]. For a network in our model, agents become nodes, and their interactions define links. We assume these mobile nodes reside in two-dimensional (2D) periodic lattices, and that node states are periodic as in phases of oscillators for ad hoc wireless networks [18]. To mimic the stochastic nature of these dynamic networks, we also assume nodes are random walkers. Then, local interactions define dynamic short-range links, which will be removed as soon as nodes move apart, while we will add long-range interactions randomly, defining long-range links. The addition of long-range interactions is justified, because they are rapidly growing in modern societies in many ways. We will refer to this network as a dynamic interaction network. In this Rapid Communication, we observe how these networks behave dynamically as the number of long-range links grows. Our model produces metastable states $[9,10,15,17]$, and we will focus on their dynamic behavior by finding their mean lifetimes.

For our model, we start with a network defined in Ref. [17] with minor modifications. It considers $N$ nodes residing on a 2D periodic lattice $(X \times Y)$ (see Fig. 1). Each node $i(1 \leqslant i \leqslant$

*suhan@physics.utexas.edu
$N)$ has its location $\left(x_{i}, y_{i}\right)$ in the lattice, where $0 \leqslant x_{i}<X$ and $0 \leqslant y_{i}<Y$. At each time step $t(=0,1,2, \ldots)$, all nodes move independently using 2D random walks, and interact with local neighbors. Links are determined by (i) how local neighbors are defined, and (ii) how nodes move on the lattice. Here we use the Moore neighborhood; hence a node is a local neighbor of node $i$ if that node resides in any of the surrounding eight locations or the current location of $i$. And the nodes move randomly in four directions (east, west, south, and north) with equal probabilities at each time step. This network is dynamic, and we will restrict ourselves to the case with $X=100, Y=10$, and $N=10^{3}$. Here we also add $E$ distinct long-range links (or shortcuts) randomly, and they are time invariant, meaning that these links will connect the same agents (nodes) all the time. The number of all possible pairs of nodes is $N(N-1) / 2$ $\left(\equiv E_{\max }\right)$, and we use a normalized value $e=E / E_{\max }(0 \leqslant$ $e \leqslant 1)$ as a control parameter. Now the network has two types of links: one representing short-range dynamic interactions, and the other representing long-range static interactions. We can use the $N \times N$ adjacency matrix $\mathbf{K}(t)$ to represent this network, where $K_{i j}(t)$ is 1 when there exists any link between $i$ and $j$ at time $t$, and 0 otherwise.

When $e=0$, the network has only short-range links, and, when $e=1$, the network is fully connected. We can look at some of the basic network properties of this network: the average degree $k(e)$, the clustering coefficient $C(e)$, and the average shortest path length $L(e)$, when all nodes are uniformly distributed on the lattice [19]. We numerically find these values by averaging over $10^{3}$ realizations: for $e=0, k(0) \simeq 8.99$, $C(0) \simeq 0.604$, and $L(0) \simeq 25.8$. When calculating $C(e)$ and $L(e)$, we only use the biggest component, if there is more than one component. In Fig. 2, we show $C(e)$ and $L(e)$ $\left[k(e) \simeq 9+10^{3} e\right.$ for $\left.e \ll 1\right]$, and we see some resemblances with the classic models of SW networks [4,5]. Here the set of $e$ can be divided into three regions: (I) $C(e)$ does not change much while $L(e)$ decreases quickly (dominated by short-range links with the $\mathrm{SW}$ phenomena); (II) $C(e)$ starts to decrease and reaches the smallest value; (III) $C(e)$ starts to increase before reaching 1 at $e=1$ (dominated by long-range links). Meanwhile, $L(e)$ monotonically decreases to 1 . The overall behavior is the same for cases with different $N$ 's. 


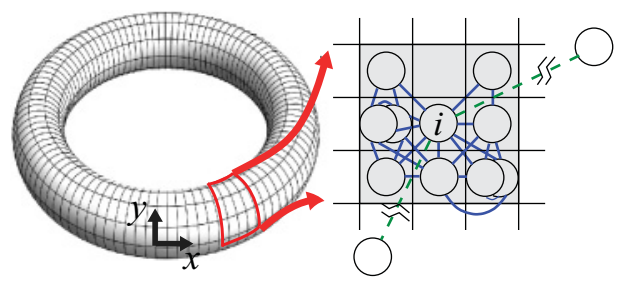

FIG. 1. (Color online) An illustration of a 2D periodic lattice. Local neighbors of node $i$ are in the shaded area, and blue (dark gray) solid lines represent dynamic short-range links, while green (light gray) dashed lines represent randomly added long-range links.

We then introduce a periodic node state $\phi_{i}\left(0 \leqslant \phi_{i}<1\right)$ to each node $i$, and observe the dynamics on the network defined above $\left(\phi_{i}\right.$ can be an opinion of an agent $i$ in opinion dynamics [17], or $2 \pi \phi_{i}$ can be a phase of one of identical oscillators in synchronization problems $[11,12,20,21])$. For changes of $\phi_{i}$ 's, we use the rule of repeated averaging as below. At time $t$, all nodes update their values in parallel using

$\phi_{i}(t+1)=\phi_{i}(t)+\frac{\sigma}{k_{i}(t)+1} \sum_{j=1}^{N} K_{i j}(t) \Delta_{j i}(t)(\bmod 1)$,

where $\sigma$ is a coupling constant $(0<\sigma \leqslant 1), k_{i}(t)$ is the degree of node $i\left(=\sum_{j} K_{i j}\right)$, and $\Delta_{j i}(t)$ represents the difference between $\phi_{j}$ and $\phi_{i}$ at time $t$. We introduce a function $\Delta(x)$ to represent the difference between two values, such that $\Delta_{j i}=$ $\Delta\left(\phi_{j}-\phi_{i}\right)$,

$$
\Delta(x) \equiv \begin{cases}x+1 & (\text { if } x<-1+\theta), \\ 0 & (\text { if }-1+\theta \leqslant x \leqslant-\theta), \\ x & \text { (if }-\theta<x<\theta) \\ 0 & \text { (if } \theta \leqslant x \leqslant 1-\theta), \\ x-1 & (\text { if } 1-\theta<x)\end{cases}
$$

where $\theta$ is a constant $(0<\theta \leqslant 0.5)$, and works as a threshold for interactions. In this Rapid Communication, we assume $\theta=0.5$ for simplicity. Note that Eq. (1) is the same as the one given in Ref. [17] with different notations.

When $e=0$, it was already found that periodic metastable states along the $x$ direction can spontaneously emerge from random initial conditions when $X \gg Y$ [17], and we call them "period- $n$ " states. When $0<e \ll 1$, they still have finite mean lifetimes $\tau_{n}^{(p)}$, which will decrease as $e$ increases because long-range links make periodic metastable states decay faster and eventually disappear. When $e$ is greater than a certain
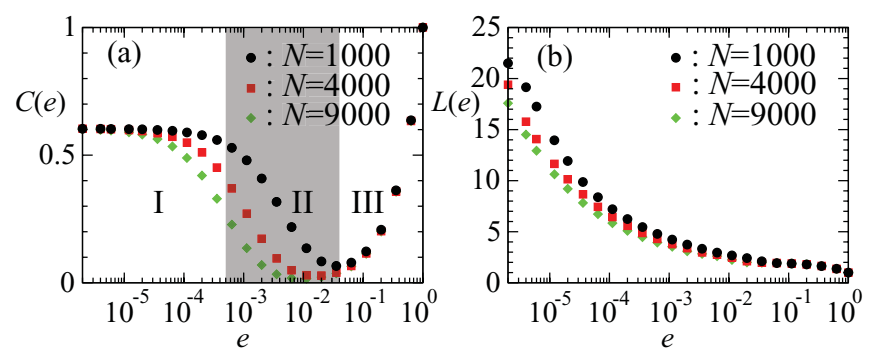

FIG. 2. (Color online) For $N$ uniformly distributed nodes when $(N, X, Y)$ is $(1000,100,10),(4000,200,20)$, and $(9000,300,30)$, we observe (a) $C(e)$ and (b) $L(e)$ vs $e$. In (a), we divide $e$ into three regions for the case of $N=10^{3}$. critical value, then period- $n$ states cannot be sustained, and only converged states, where all node states are the same, will be reached. As $e$ increases further, however, other types of metastable states start to appear; they are grouping metastable states, where nodes are divided into $m$ separate groups of clustered states, independent of their positions on the lattice. For these states, the number of nodes in groups and the differences in the states between the groups should satisfy certain conditions: for example, three groups with similar sizes near $0,1 / 3$, and $2 / 3$, respectively, or $\sim 500$ nodes near 0.5 , and two other groups of $\sim 250$ nodes near 0.1 and 0.9 , respectively. We call them " $m$-group" states [22]. Their mean lifetimes increase as $e$ increases, and as $e$ approaches 1, $m$-group states become more stable. Meanwhile, converged states are absorbing states in every case (consensus in opinion dynamics, or synchronized states in synchronization problems). If either the node state or the lattice is not periodic, the system only reaches converged states with repeated-averaging mechanisms, while $e$ and $\sigma$ only determine the convergence time.

Here we numerically study period- $n$ states in detail, as well as 3 -group states (the smallest possible here). Usually, period- $n$ states decay into period- $(n-1)$ states, and later into period$(n-2)$ states, until they eventually reach converged statesthis is a decay chain. To investigate this decay process closely, one needs a way to find what period a given state is in. We introduce $v$ for this purpose. First, we divide the lattice into $N_{r}$ equal-sized regions along the $x$ direction. (We assume $X$ is a multiple of $N_{r}$, and use $N_{r}=50$ here.) Second, for each region $r\left(0 \leqslant r<N_{r}\right)$, we find the average of node states for nodes inside that region, $\bar{\phi}_{r}$. Since the node state is periodic, care has to be taken; for example, the average of 0.1 and 0.9 should be 0 instead of 0.5 [Eq. (2) should be used when averaging]. Once we find all $\bar{\phi}_{r}$ 's for all $r$, we can finally define $v$ as

$$
v \equiv\left|\sum_{r=0}^{N_{r}-1} \Delta\left(\bar{\phi}_{r+1}-\bar{\phi}_{r}\right)\right|,
$$

where $\bar{\phi}_{N_{r}}=\bar{\phi}_{0}$. For period- $n$ states, $v$ is close to $n$, and we can observe the behavior of a state by observing the time evolution of $\nu$.

In Fig. 3, we observe how a random state evolves for different $e$ 's, when $\sigma=0.1$. When $e<0.001$, the state quickly becomes a period-2 state, and it does not decay even at $t=$ 30000 . But when $e=0.001$, we can observe the period- 2 state decays into a period- 1 state, and then becomes a converged state. Note that the lifetimes found here are only for this run, and the mean lifetimes for $e=0.001$ are shown in Fig. 4. As $e$ increases further, period- $n$ states tend to decay faster, and for higher $e$ 's, period- $n$ states are reached only for a short time or bypassed (when $e=0.002$, the state tries to reach a period-1 state, but fails before becoming a converged state).

When $\sigma$ is not too small, the state quickly reaches any period- $n$ state or a converged state given an initial condition, and distributions of these states depend on all parameters [17]. When mean lifetimes are short enough to be measured, these distributions visibly change as time evolves, and we can predict that the ratio of the number of converged states to the number of all runs at time $t, \rho_{0}(t)$, will eventually reach 1 in a reasonable time. Decays from $n$ to $n-1$ are exponential, which means that, if we specifically choose an initial condition as a period- $n$ 


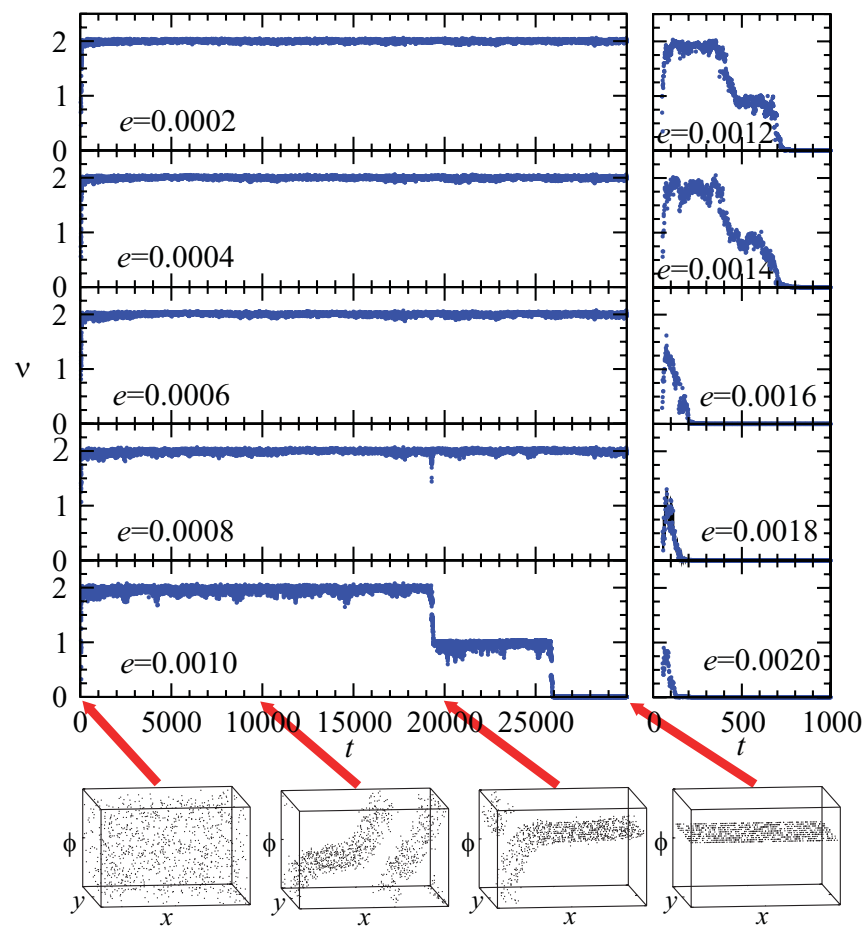

FIG. 3. (Color online) Time evolution of $v$ for a state as $e$ increases from 0.0002 to 0.002 when $\sigma=0.1$. Bottom: Scatter plots for $e=0.001$ at $t=0$ (random initial condition), 10000 (period 2), 20000 (period 1), and 30000 (converged).

state and observe the ratio of period- $n$ states among many runs at time $t$, we can find $\tau_{n}^{(p)}$ using the method of linear least squares. In Fig. 4 , we find $\tau_{n}^{(p)}(1 \leqslant n \leqslant 5)$ and $\tau_{3}^{(g)}$, the mean lifetime of states with three equal-sized groups, as $e$ varies when $\sigma=1$ and 0.1 . We found several interesting properties for $\tau_{n}^{(p)}$ here. For period- $n$ states for low $n$, there exists a critical $e$ value, $e_{n}^{(p)}$, after which period- $n$ states cannot be sustained-for example, $e_{1}^{(p)} \simeq 0.002$ when $\sigma=0.1$ (see Fig. 3). When $e>e_{n}^{(p)}$, period- $n$ states cannot be sustained for even a short period of time, while the variability of $\tau_{n}^{(p)}$ with different realizations becomes greater. When $e<e_{n}^{(p)}$, $\tau_{n}^{(p)}$ does not depend much on initial conditions as long as they are period- $n$ states. Higher-period states can be more stable than lower-period ones for certain $e$ 's. On the other hand, when $e$ gets close to 1 , grouping metastable states can exist. The insets in Figs. 4(a) and 4(b) prove their existence: when $e>0.1, \rho_{0}$ can stay less than 1 for a considerable amount of time. The closer $e$ is to 1 , the longer the mean lifetimes of these metastable states become.

In Fig. 4(c), we observe $\tau_{1}^{(p)}$ and $\tau_{3}^{(g)}$ for the whole range of $e$ when $\sigma=1$ and 0.1 . There also exists a critical value $e_{3}^{(g)}$ for 3-group states. When $e>e_{3}^{(g)}, 3$-group states can exist and $\tau_{3}^{(g)}$ increases with $e$. When $e<e_{3}^{(g)}, 3$-group states cannot be sustained. Based on the results for $\sigma=1$, we divide $e$ into three regions, which match well with those found in Fig. 2(a): (I) $0<e<e_{1}^{(p)}$, where periodic metastable states can exist (dominated by short-range links); (II) $e_{1}^{(p)}<e<e_{3}^{(g)}$, where only converged states are sustained; (III) $e_{3}^{(g)}<e \leqslant 1$,
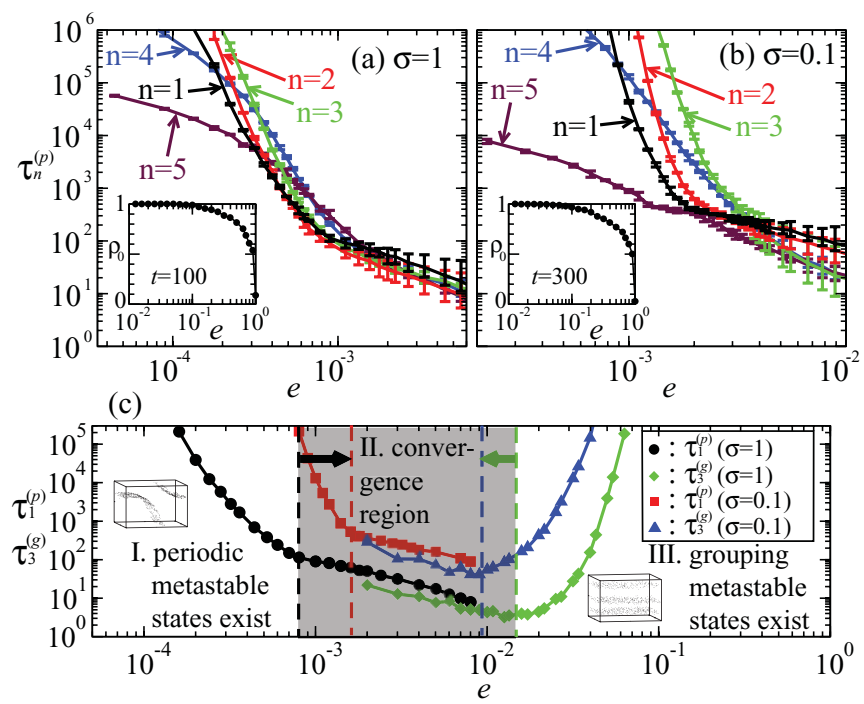

FIG. 4. (Color online) Behavior of $\tau_{n}^{(p)}(1 \leqslant n \leqslant 5)$ and $\tau_{3}^{(g)}$ as $e$ varies. (a) $\tau_{n}^{(p)}$ when $\sigma=1$, (b) $\tau_{n}^{(p)}$ when $\sigma=0.1$. For each parameter set, we used three different realizations of period- $n$ states with $10^{3}$ runs each. The bars represent minimum and maximum values. (The insets show ratios of the converged states, $\rho_{0}$, after a certain time from $10^{3}$ random initial conditions, when $e$ approaches 1 , to show that grouping metastable states appear spontaneously.) (c) $\tau_{1}^{(p)}$ and $\tau_{3}^{(g)}$ for $0<e \leqslant 1$ when $\sigma=1$ and 0.1 . There are three regions of $e$, and the middle region is shaded for the case of $\sigma=1$.

where grouping metastable states can exist (dominated by long-range links). It is also interesting that the range of $e$, where small-world phenomena are observed, coincides with the range where periodic metastable states have measurable mean lifetimes. We also observe results for $\sigma=0.1$, and they show similar behavior with longer mean lifetimes and a smaller convergence region. If $\sigma$ is much smaller, the system can behave in an unexpected manner; for example, even when $e=0$, grouping metastable states can appear (see Fig. 8 in Ref. [17]).

In summary, we studied the dynamics of random-walking agents with periodic node states in a periodic lattice, varying the number of long-range links, and observed two types of metastable states in the system. Metastable states in our model can be interpreted as dynamic, yet sustainable, diversities abundant in our societies [23], and results imply that the ever-increasing connections of our modern societies can make existing diversities disappear, but if we are almost fully connected, other types of diverse states can appear under this rule.

We can ask if specific settings we assumed in the model are all necessary to observe similar behavior. What if we apply the same dynamic rule on static SW networks on one-dimensional (1D)-ring structures with $E$ long-range links, as the model by Newman and Watts [5]? Without random rewiring of short-range links, there is no stochasticity, and we can find fixed points instead of metastable states. While period- $n$ states are apparently stable when $E=0$, other nonconverged states are dominant when $E$ is small (see Ref. [24] for details). If we go even further, we can say that as long as the network is sparse and cyclic, nonconverged states can be spontaneously reached. As the network becomes almost fully connected, 
where underlying structures and short-range links do not matter much, grouping states become stable under this rule, too. What if we change the update rule represented by $\Delta(x)$ ? It is easy to see that period- $n$ states will be stable in similar networks as long as $\Delta(x) \simeq x$ near $x=0$. For grouping states to be stable for $e \rightarrow 1$, though, the slope of $\Delta(x)$ should be positive or zero throughout the whole range of $x$ as in Eq. (2). For example, if we use $\Delta(x) \propto \sin (2 \pi x)$, the model is basically the Kuramoto model [25] for identical oscillators; in this case, grouping states cannot be stable even at $e=1$, while period- $n$ states can be stable at $e \simeq 0$.
In addition, we can extend this model to suit realistic needs. A coupling constant $\sigma$ is given to both short- and long-range interactions here, but we can have two coupling constants [12], or it can be heterogeneous among all nodes. We can add noise in Eq. (1), because noise can play an important role in clustering [23]. Long-range links can be rewired, making the model truly coevolving.

The author would like to thank L. E. Reichl for useful discussions, and Kongju National University for financial support.
[1] R. Albert and A.-L. Barabási, Rev. Mod. Phys. 74, 47 (2002).

[2] S. Boccaletti, V. Latora, Y. Moreno, M. Chavez, and D.-Y. Hwang, Phys. Rep. 424, 175 (2006).

[3] S. N. Dorogovtsev, A. V. Goltsev, and J. F. F. Mendes, Rev. Mod. Phys. 80, 1275 (2008).

[4] D. J. Watts and S. H. Strogatz, Nature (London) 393, 440 (1998).

[5] M. E. J. Newman and D. J. Watts, Phys. Rev. E 60, 7332 (1999).

[6] A.-L. Barabási and R. Albert, Science 286, 509 (1999).

[7] M. Barahona and L. M. Pecora, Phys. Rev. Lett. 89, 054101 (2002).

[8] M. F. Laguna, G. Abramson, and D. H. Zanette, Physica A 329, 459 (2003).

[9] K. Suchecki, V. M. Eguíluz, and M. San Miguel, Phys. Rev. E 72, 036132 (2005).

[10] R. Toivonen, X. Castelló, V. M. Eguíluz, J. Saramäki, K. Kaski, and M. San Miguel, Phys. Rev. E 79, 016109 (2009).

[11] H. Hong, M. Y. Choi, and B. J. Kim, Phys. Rev. E 65, 026139 (2002).

[12] R. Heylen, N. S. Skantzos, J. Busquets Blanco, and D. Bollé, Phys. Rev. E 73, 016138 (2006).

[13] P. Holme and M. E. J. Newman, Phys. Rev. E 74, 056108 (2006).

[14] M. C. González, P. G. Lind, and H. J. Herrmann, Phys. Rev. Lett. 96, 088702 (2006).
[15] I. J. Benczik, S. Z. Benczik, B. Schmittmann, and R. K. P. Zia, Phys. Rev. E 79, 046104 (2009).

[16] S. C. Manrubia, J. Delgado, and B. Luque, Europhys. Lett. 53, 693 (2001).

[17] S. Ree, Phys. Rev. E 83, 056110 (2011).

[18] N. Fujiwara, J. Kurths, and A. Díaz-Guilera, Phys. Rev. E 83, 025101 (2011).

[19] Because the motion of nodes is diffusive, the density of the nodes on the lattice converges to the uniform distribution regardless of the initial positions of the nodes.

[20] A. Arenas, A. Díaz-Guilera, J. Kurths, Y. Moreno, and C. Zhou, Phys. Rep. 469, 93 (2008).

[21] A. Díaz-Guilera, J. Gómez-Gardeñes, Y. Moreno, and M. Nekovee, Int. J. Bifurcat. Chaos 19, 687 (2009).

[22] Even-numbered groups cannot be stable when $\theta=0.5$ in this setting, because there exist groups with differences at 0.5 , where the $\Delta$ function changes the sign abruptly.

[23] M. Mäs, A. Flache, and D. Helbing, PLoS Comput. Biol. 6, e1000959 (2010).

[24] S. Ree, e-print arXiv:1203.3241 (2012).

[25] Y. Kuramoto, Chemical Oscillations, Waves and Turbulence (Springer, New York, 1984); J. A. Acebrón, L. L. Bonilla, C. J. Pérez Vicente, F. Ritort, and R. Spigler, Rev. Mod. Phys. 77, 137 (2005). 